\title{
Fully Differential Electro-Mechanical Phase Locked Loop Sensor Circuit
}

\author{
Robert Rudolf, Peter R. Wilson, Li Ke, Reuben Wilcock, An- \\ drew D. Brown and Nick R. Harris \\ School of Electronics and Computer Science, University of Southampton, Southampton, \\ UK, SO171BJ e-mail: prw@ecs.soton.ac.uk
}

\begin{abstract}
Embedding a micro-machined sensing element in a closed loop, force feedback system is a technique commonly used to realise high performance MEMS (micro-electro-mechanical systems) sensors due to the advantages of better linearity, increased dynamic range and reduced parameter sensitivity. Electro-mechanical Sigma Delta modulators $(\operatorname{EM} \Sigma \Delta)$ have been proposed for this reason and high order loops have been shown to enjoy a good signal to noise ratio (SNR) of more than $100 \mathrm{~dB}$. It is also well known that achieving stability in high order $\operatorname{EM} \Sigma \Delta \mathrm{s}$ is a challenging task and in practice stability can be lost with large input signals or due to non-ideal effects in the circuits implemented. In this work we propose a novel differential frequency domain technique for closed loop control of micro-machined sensors. This method, called the electro-mechanical phase locked loop (EMPLL), uses a differential electro-mechanical phase locked loop to control and measure the deflection of micro-machined sensors. We believe that EMPLLs have the potential to have significant advantages over $\operatorname{EM} \Sigma \Delta$ s for high performance MEMS sensors. Preliminary research suggests that this novel approach will
\end{abstract}


lead to significant benefits in Signal to Noise Ratio, Parameter Sensitivity, and Input Signal Range.

Keywords: Phase Locked Loop, Capacitive Sensor Circuit, MEMS Sensor

\section{Introduction}

The use of Electro-Mechanical Sigma Delta Modulators $(\operatorname{EM} \Sigma \Delta)$ for high performance sensors in multiple applications has become pervasive over the last decade or so. One issue for designers of integrated electronic and MEMS circuits has been that while the EM $\Sigma \Delta$ approach offers generally good Signal to Noise Ratio (SNR), of the order of $100 \mathrm{~dB}$, this is often at the price of stability. It is also notoriously difficult to design stable and robust higher order Sigma Delta modulators. Another design constraint is that the input signal range tends to be limited due to the intrinsic tendency of the sigma delta modulator to become unstable for higher signal levels. In practical integrated systems, the overall design stability is very sensitive to parameter variation. Whether the variation is induced by environmental changes (such as temperature) or degradation over time, the circuits tend to be difficult to design with inherent robustness. This is a particular problem even with optimized parameter sets, as a nominal parameter set is often very sensitive to very small parameter changes.

Various options for improving these aspects of $\operatorname{EM} \Sigma \Delta \mathrm{s}$ have led to a significant research effort in the area of parameter optimization and architecture design, however there is a tension ultimately between the high orders required to achieve good SNR and the resulting parameter sensitivity. The primary goal of this research has therefore been to identify a potential al- 
ternative electronic interface circuit to the conventional $\operatorname{EM} \Sigma \Delta$ that could provide equivalent SNR performance, but would potentially be easier to design and offer better dynamic range and increased tolerance to parameter variation. This paper will use the standard principles of force feedback capacitive sensors, and offer an alternative to the standard $\operatorname{EM} \Sigma \Delta$ Modulator approach. A new fully differential technique for sensing changes in capacitive sensors will be introduced and the work will demonstrate how this can be used in a force-feedback control loop. The new approach is compared with a typical $5^{\text {th }}$ order $\operatorname{EM} \Sigma \Delta$ Modulator to give an indication of the relative merits of the two methods.

\section{Background}

It has become common practice to include both a Micro-Electro-Mechanical System (MEMS) sensor with an electronic sensor interface circuit, using a closed loop approach where the sensor itself is part of the control loop. MEMS inertial sensors are often based on a capacitive sensing element, and do have an advantage when linked to a $\Sigma \Delta$ modulator in that they provide a digital output that can be connected directly to a digital circuit for further processing. Using a MEMS sensor with a lower order $\Sigma \Delta$ modulator provides second order systems that are simple to design $[1,2]$, relatively stable and have reasonable performance. Unfortunately, the inherent disadvantages of lower order $\Sigma \Delta$ modulators in the electronic domain are also well known and manifest themselves in the integrated MEMS sensor type, including quantization noise, dead-zones and the issue of idle tones becoming apparent in the signal bandwidth. 
With the increasing requirement for sensitivity and low noise, the basic approach has been to develop higher order $\Sigma \Delta$ modulators, with an integrated EM (Electro Mechanical) Sensor in the loop, with $5^{\text {th }}$ order systems providing excellent Signal to Noise ratio and overall performance [3, 4]. In spite of these efforts, it has become necessary to provide advanced optimization tools [5] to establish the correct $\Sigma \Delta$ design parameters to ensure stability. While this type of approach can calculate the nominal optimum parameters for a circuit, in practice these can be extremely sensitive to component variation. Even if the parameters are designed to be more robust to variation, as suggested in [5], this involves a complex and time consuming optimization process. This research therefore provides insight into the possibility of using a Phase Locked Loop (PLL) sensing circuit rather than $\Sigma \Delta$ based modulator, to establish whether there would be any potential advantages which would alleviate the sensitivity and difficulty in obtaining effective and robust design parameters. The method of applying the principles of a phase-locked loop to a differential force-balanced accelerometer interface circuit has not been previously described in literature. There are previous methods that use parts of this idea, but none show a combination of all three key elements (phase or frequency-based, differential and closed-loop) at the same time.

Matsumoto [6] describes a single ended PLL system where the VCO contains the variable capacitor of the sensor. This means that the PLL will run normally and locked to a reference, but acceleration on the sensor causes an "error" in the VCO frequency which is compensated by changing the VCO input voltage. This system, however, works on a single-ended sensor capacitance and has no electromechanical feedback to the sensor. Matsumoto 
[7] also presented a system which uses a PLL using the sensor as part of a variable-frequency oscillator with the feedback voltage as an input. The capacitor driving the oscillator is also single-ended, with a separate reference capacitor on the MEMS chip. Key differences to the proposed EMPLL are firstly that the capacitance is not differential, but instead use a fairly complex and precision-limited periodically updated counter to obtain the reference frequency for the PLL from a fixed reference on the sensor chip. Secondly the feedback system is also single-ended and therefore the feedback plates are not biased at a certain voltage and finally the loop topology in Matsumoto's system is quite different from the EMPLL, mainly due to the particular implementation of the oscillators.

Kitano et al [8] use a differential sensor that drives two independent LC oscillators to account for errors from drift and parasitics. They suggest that the potential exists to tune the oscillators however there is no feedback to the mechanical system. In a system for resonant gyroscopes presented by Saukoski [9], there are two control loops, one to drive the resonator and another one for compensation. However, although the resonator part looks like a PLL, this approach does not utilize a frequency that is generated from a variable oscillator. There are numerous other pickoff systems for gyroscopes that work in a very similar fashion such as [10]. Partridge et al [11] describe MEMS resonators of which they measure the resonance frequency, which changes under acceleration. They present a number of sensing circuits and also provide feedback to the mechanical system that is generated by an unspecified "Control Circuitry". Although they use PLLs to measure the frequency, the feedback is not directly within the PLL control loop - they 
merely use it to measure the frequency, among other approaches. Hati et al [12] use stable PLLs and investigate the effects of vibration on frequency references. Although there is no electromechanical feedback as the topology is essentially the same as [6], it is still relevant because of the analysis of closed-loop system behaviour. Yoneoka et al [13] look at random vibration noise in resonant systems and how it could be compensated, also by using a dedicated accelerometer. Again, there is no electromechanical feedback or indeed a full pickoff circuit, but this work is nevertheless relevant for noise considerations.

In summary, therefore, the basic idea of using a Phase Locked Loop with the capacitive sensor providing some basic control of the oscillator frequency in the loop has some precedent, however the use of a fully differential system has not been described in the literature.

\section{The Electro-Mechanical Phase Locked Loop (EMPLL)}

In a conventional Electro-Mechanical Capacitive Sensor circuit the variation in capacitance directly modulates the signal applied to the sensor (usually a high frequency "carrier" signal). This is demodulated by a $\Sigma \Delta$ circuit to generate a digital output and also provide a suitable force feedback signal to the sensor to keep it under control, and the system "in lock". In this section, we introduce the EMPLL concept, which is different in that unlike the electromechanical $\Sigma \Delta$ modulator, which measures sensor capacitance directly by means of a charge amplifier, the electromechanical PLL architecture shown in 1 uses a pair of oscillators with the sensor capacitance as the frequency determining element. 


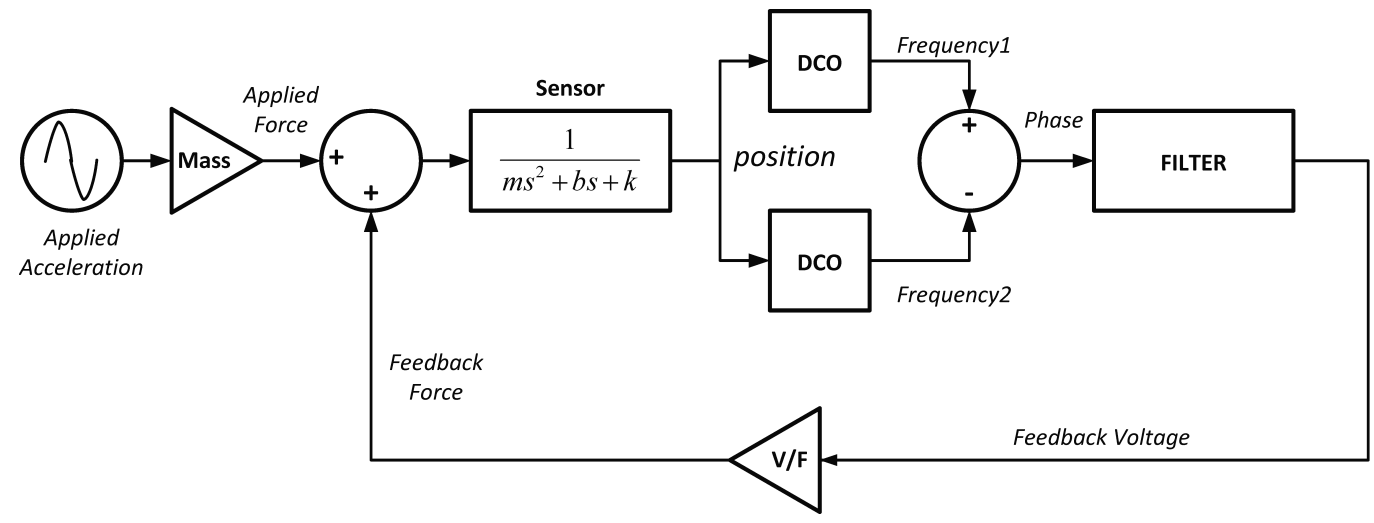

Figure 1: Architecture of the EMPLL.

The two sensor capacitances determine the frequency of two oscillators, which are labelled DCO (displacement-controlled oscillator) in Figure 1. The name displacement-controlled oscillator is in reference to a voltage-controlled oscillator (VCO), but indicates that the oscillation frequency is determined by the displacement of the accelerometer's proof mass rather than a control voltage. Since the capacitors change differentially when the sensor is subject to acceleration, the oscillator frequencies change likewise. The difference in frequency between the two oscillators is thus a measure for the input acceleration. This principle is illustrated in Figure 2. The outputs of the oscillators are input to a phase detector and a loop filter to suppress the "carrier" and provide a feedback control signal.

Effectively, this entire system behaves like a phase-locked loop, where one of the sensor oscillators represents the reference oscillator and the second oscillator is equal to the feedback controlled VCO. In this configuration, the EMPLL shows several advantages over $\Sigma \Delta$ modulators or conventional 


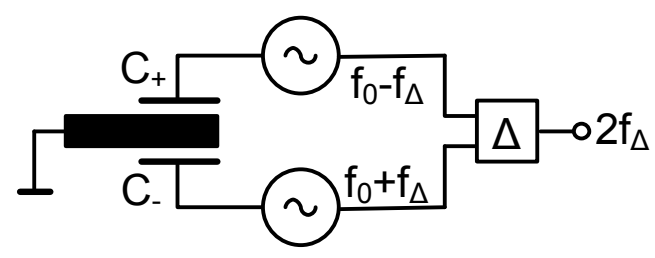

Figure 2: Frequency difference as a measure for acceleration.

pick-off circuits. In the first place, no linear low-noise charge amplifier is required, which simplifies the circuit configuration. In contrast to the $\Sigma \Delta$ System, the order does not need to be increased to gain SNR, and this has the secondary effect of making the system performance inherently more robust against parameter variation. Finally, there is no digital switching of feedback voltage, which has a positive impact on the noise in the system. The most important fact about the DCO to note is that ideally the difference in frequency is linearly dependent on acceleration, which is shown below. It should be noted that there is a well defined range of operation for any controlled oscillator and outside this range a practical system will limit the frequency of the oscillator - potentially introducing a non-linearity. In a practical system, therefore, the oscillators should be designed with the centre frequency and sensitivity to match the range of operation of the sensor. The force $(F)$ experienced by the proof mass $(m)$ in the accelerometer under acceleration $(a)$ is:

$$
F=m a
$$

and the resulting displacement of the proof mass is that of a damped 
second-order mass-spring-damper system:

$$
d=\frac{1}{m s^{2}+b s+k} F=k_{\text {sens }}(s) a
$$

with

$$
k_{\text {sens }}=\frac{m}{m s^{2}+b s+k}
$$

where $m$ is the proof mass, $b$ is the damping factor, $k$ the spring constant of the mass-spring system that models the accelerometer and $s$ is a complex number in the Laplace space. Recalling the generic capacitance of a parallel plate capacitor as an approximation for the sensing capacitance:

$$
C=\epsilon_{0} \epsilon_{r} \frac{A}{d}=k_{c a p} \frac{1}{d}
$$

with

$$
k_{\text {cap }}=\epsilon_{0} \epsilon r A
$$

where $\mathrm{A}$ is the sensor capacitor plate area and $d$ the spacing between the plates. Substituting the instantaneous displacement $(d)$ from equation 2 around the nominal capacitor plate spacing (dnom), the sensor capacitance can be written as a function of acceleration $(a)$.

$$
C=\epsilon_{0} \epsilon_{r} \frac{A}{d_{\text {nom }} \pm d}=k_{\text {cap }} \frac{1}{d_{\text {nom }} \pm k_{\text {sens }} a}
$$

It is desirable to have a linear relationship between frequency and acceleration, which requires the oscillator frequency to be inversely proportional to capacitance making an RC oscillator the ideal choice. An LC oscillator would generally be the first choice from the point of view of low noise design, 
however the frequency of such an oscillator is inherently dependent on the inverse root of the capacitance.

$$
f_{\text {osc }}=k_{\text {osc }} \frac{1}{C}
$$

The parameter $k_{\text {osc }}$ relates the frequency to capacitance, depending on resistance values and switching thresholds. As can be see in equation 6, however, the capacitance is not exclusively dependent on the inverse of the acceleration, but also on the nominal capacitor plate spacing $d_{\text {nom }}$. However, this constant term, which may well be larger in magnitude than the change in capacitance due to acceleration, cancels when two oscillators are driven from a pair of differential capacitors (in the symmetrical structure shown in 1) and are subject to the same acceleration. The frequency difference of two such oscillators is:

$$
\begin{array}{r}
\Delta f=f_{+d}-f_{-d}=\frac{k_{o s c}}{k_{\text {cap }}}\left(d_{\text {nom }}+d-d_{\text {nom }}+d\right) \\
\Delta f=2 \frac{k_{\text {osc }}}{k_{\text {cap }}} d
\end{array}
$$

As we have seen in Equation 2, the displacement can be replaced with a function of acceleration and as a result the frequency difference can be seen in 9 to be linear with respect to acceleration.

$$
\Delta f=2 \frac{k_{\text {osc }}}{k_{\text {cap }}} k_{\text {sens }}(s) a
$$

The output voltage proportional to acceleration cannot be applied directly to the feedback plates. The reason for this is that the electrostatic 
force between the two feedback plates is only attractive, regardless of the polarity of the feedback voltage. It is therefore necessary to apply a certain bias voltage to both feedback plate pairs, causing oppositely directed forces on the proof mass resulting in no net force when under quiescent conditions. The feedback voltage is then superimposed on this bias voltage, resulting in an increase of one and decrease of the other force, resulting in a net feedback force on the proof mass, as depicted in Figure 3.

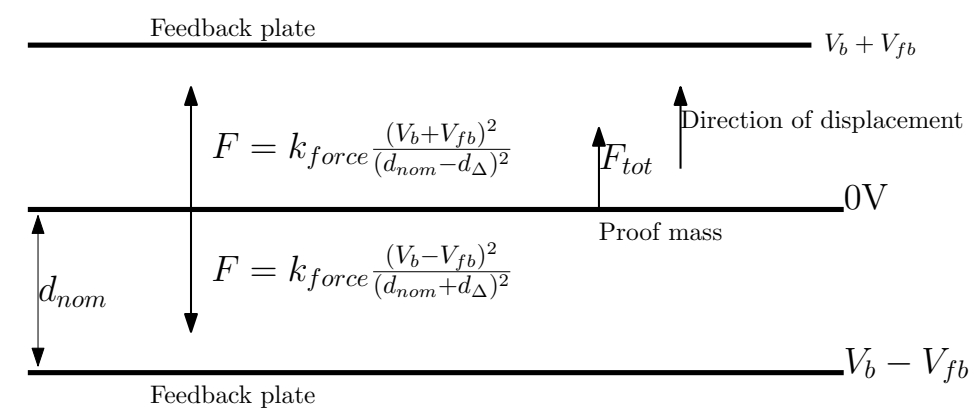

Figure 3: Forces in the force-feedback system.

Generally, the electrostatic force between two parallel plates is given by Eq. 10.

$$
F=k_{\text {force }} \frac{V^{2}}{d^{2}}
$$

where:

$$
k_{\text {force }}=\frac{1}{2} \epsilon_{0} \epsilon_{r} A
$$

The total force on the proof mass is the sum of two oppositely directed electrostatic forces, each depending on the bias voltage and the differential feedback voltage as given in Eq. 12. 


$$
\begin{array}{r}
F=k_{\text {force }}\left(\frac{\left(V_{b}+V_{f b}\right)^{2}}{\left(d_{n o m}-d_{\Delta}\right)^{2}}-\frac{\left(V_{b}-V_{f b}\right)^{2}}{\left(d_{n o m}+d_{\Delta}\right)^{2}}\right) \\
F=k_{\text {force }}\left(\frac{V_{b}^{2}+2 V_{b} V_{f b}+V_{f b}^{2}}{\left(d_{\text {nom }}-d_{\Delta}\right)^{2}}-\frac{V_{b}^{2}-2 V_{b} V_{f b}+V_{f b}^{2}}{\left(d_{\text {nom }}+d_{\Delta}\right)^{2}}\right)
\end{array}
$$

Due to the dependence of the force on the instantaneous spacing of the plates, the relationship between feedback force and feedback voltage is not linear. Assuming higher order effects can be ignored due to the small displacements involved, Eq. 14 shows that the feedback force is approximately linearly dependent on feedback voltage.

$$
F \approx k_{\text {force }} \frac{4 V_{b} V_{f b}}{d_{n o m}^{2}}
$$

This result means that the entire closed-loop system of the EMPLL is approximately linear, which is a very significant result. Unlike electromechanical $\Sigma \Delta$ modulators which are inherently non-linear and therefore require difficult analysis methods, initial design calculations and simulations on the EMPLL can be achieved using a linear approximation. Once the system properties and parameters have been determined roughly using the linear approximation, final tuning can be conducted using the full non-linear models, which also give the most accurate performance metrics.

As an example of this it can be seen from equation 12 that in addition to non-linearity introduced by the instantaneous displacement, the feedback voltage also appears as a squared term in the numerator. This causes distortion of the signal, which can be seen in the simulations as a harmonic component of twice the fundamental frequency, as in $\Sigma \Delta$ systems. For $\Sigma \Delta$ systems, an adaptive feedback voltage is usually used to reduce this harmonic 
component. In the EMPLL, however, reduction of this component can be achieved more easily and without any additional circuitry. If the feedback plate bias voltage is increased to be relatively large with respect to the feedback voltage, the squared term of the feedback voltage becomes insignificant relative to the constant bias voltage and the linear term.

\section{Comparison of EMPLL and EM $\Sigma \Delta$ Systems}

\subsection{Introduction}

In order to evaluate the performance of the proposed approach, three standard tests have been used to compare the new EMPLL circuit with a reference $\operatorname{EM} \Sigma \Delta$ system. The first test is to calculate Power Spectral Density (PSD) of both the EMPLL and EM $\Sigma \Delta$ circuits. The main criteria at this stage is to establish whether the basic performance is comparable between the two approaches. The second test was to compare the response of the two circuits to a wide range of accelerations, and therefore the sensor and circuit combinations were tested up to $15 \mathrm{~g}$, which was the designed range of operation of the sensor. The final test was to evaluate the impact of parameter variations on the two circuit configurations, using equivalent basic parameter tolerances to see how well the two alternative approaches operated.

A selection of simulation results is presented which illustrate the performance and properties of the EMPLL. All simulations were conducted using models in Matlab and the system parameters were optimized using the Cheetah GA system [5]. In order to provide a comparison to existing methods, a 5 th order $\Sigma \Delta$ based modulator system was analysed as a representative reference circuit. 


\begin{tabular}{|l||l|l|l|l|}
\hline Parameter & Symbol & Value & Unit & Variation \\
\hline \hline Proof mass & $m$ & $1.23 \cdot 10^{-6}$ & $\mathrm{~kg}$ & $2 \%$ \\
\hline Damping coefficient & $b$ & $9.0 \cdot 10^{-4}$ & & $25 \%$ \\
\hline Spring constant & $k$ & 67 & $N \cdot m^{-1}$ & $5 \%$ \\
\hline Plate spacing & $d_{\text {nom }}$ & $6.5 \cdot 10^{-6}$ & $m$ & \\
\hline Sense plate area & area & $2.85 \cdot 10^{-6}$ & $\mathrm{~m}^{2}$ & \\
\hline Feedback plate area & fb_area & $1.06 \cdot 10^{-6}$ & $\mathrm{~m}^{2}$ & \\
\hline
\end{tabular}

Table 1: Sensor parameters

Table 1 lists the sensor parameter used for the simulation of both the $\operatorname{EM} \Sigma \Delta$ and the EMPLL, Table 2 lists the system values used for the simulation of the $\operatorname{EM} \Sigma \Delta$ and Table 3 lists the system values used for the simulation of the EMPLL. The variations indicated are used for the simulations in Section 4.4 .

\subsection{Output Power Spectrum}

As in most sensor interface systems, the output voltage PSD is of most interest to compare system performance - especially when a $\Sigma \Delta$ approach is employed as one of the key advantages is the ability to shape the noise and achieve a significantly improved noise floor. The reference system was tested and the PSD of the reference $\operatorname{EM} \Sigma \Delta$ system from a time-domain simulation with an input amplitude of $2.5 \mathrm{~g}$ and an input frequency of $32 \mathrm{~Hz}$ is shown in Figure 4a. In this reference system the simulated SNR was 108.5dB for a signal bandwidth of $1024 \mathrm{~Hz}$ (it should be noted that the pick-off amplifier was modelled as noiseless, so the source of noise was discretization only). 


\begin{tabular}{|l||l|l|l|l|}
\hline Parameter & Symbol & Value & Unit & Variation \\
\hline \hline Compensator pole & pole & $1.57 \cdot 10^{6}$ & $s^{-1}$ & $5 \%$ \\
\hline Compensator zero & zero & $2.98 \cdot 10^{4}$ & $s^{-1}$ & $5 \%$ \\
\hline Pickoff amplifier gain & $k_{p o}$ & $4 \cdot 10^{5}$ & & $5 \%$ \\
\hline Boost amplifier gain & $k_{b s t}$ & 255.6 & & $2 \%$ \\
\hline Forward gain 1 & $k_{1}$ & 1.114 & & $2 \%$ \\
\hline Forward gain 2 & $k_{2}$ & 0.302 & & $2 \%$ \\
\hline Forward gain 3 & $k_{3}$ & 0.665 & & $2 \%$ \\
\hline Feedback gain 1 & $k f_{1}$ & 0.293 & & $2 \%$ \\
\hline Feedback gain 2 & $k f_{2}$ & 0.898 & & $2 \%$ \\
\hline Feedback gain 3 & $k f_{3}$ & 0.581 & & $2 \%$ \\
\hline Feedback voltage & $v_{f b}$ & 18.20 & $V$ & $2 \%$ \\
\hline Force feedback linearisation & $k_{f f l}$ & 7.55 & & $2 \%$ \\
\hline
\end{tabular}

Table 2: $\operatorname{EM} \Sigma \Delta$ system parameters 


\begin{tabular}{|l||l|l|l|l|}
\hline Parameter & Symbol & Value & Unit & Variation \\
\hline \hline Lead filter pole & $p_{\text {lead }}$ & $6.43 \cdot 10^{5}$ & $s^{-1}$ & $5 \%$ \\
\hline Lead filter zero & $z_{\text {lead }}$ & $1.47 \cdot 10^{4}$ & $s^{-1}$ & $5 \%$ \\
\hline Lag filter pole & $p_{\text {lag }}$ & 232.84 & $s^{-1}$ & $5 \%$ \\
\hline Lag filter zero & $z_{\text {lag }}$ & $7.54 \cdot 10^{3}$ & $s^{-1}$ & $5 \%$ \\
\hline Bias voltage & $V_{\text {bias }}$ & 18.5 & $V$ & $5 \%$ \\
\hline Compensator gain & $k_{\text {comp }}$ & 0.95 & & $5 \%$ \\
\hline Oscillator gain & $k_{D C O}$ & $4.25 \cdot 10^{11}$ & $H z \cdot m^{-1}$ & $5 \%$ \\
\hline
\end{tabular}

Table 3: EMPLL system parameters

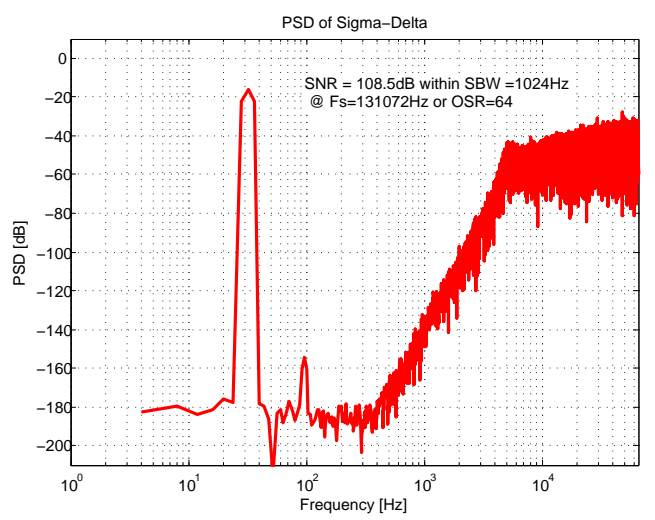

(a) PSD of $\operatorname{EM} \Sigma \Delta$

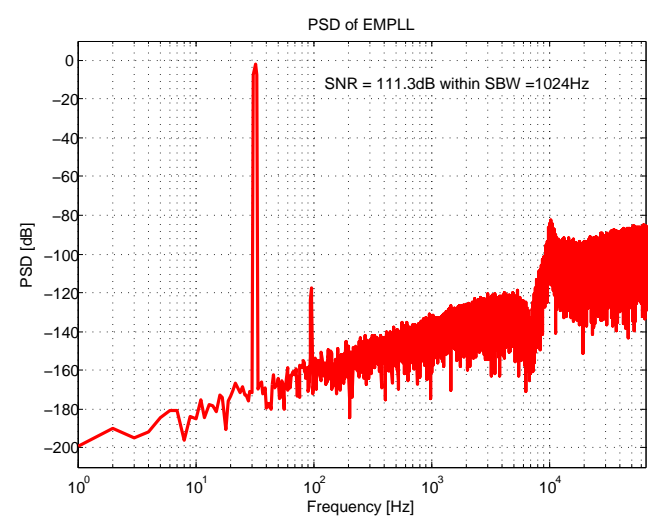

(b) PSD of EMPLL

Figure 4: Comparison of PSD between $\operatorname{EM} \Sigma \Delta$ and EMPLL 
The EMPLL system was tested in the same manner as the reference $\operatorname{EM} \Sigma \Delta$ system and the PSD obtained from a time-domain simulation with an input amplitude of $2.5 \mathrm{~g}$ and an input frequency of $32 \mathrm{~Hz}$ is shown in Figure 4b. From this PSD plot, the simulated SNR of the output signal was $111.3 \mathrm{~dB}$, which in this case is slightly better than the reference system.

It has to be noted that the presence of the harmonic component at $96 \mathrm{~Hz}$ is clearly visible and measurable. As discussed in Section 3, this harmonic results from the non-linearity in the feedback system and can be reduced by increasing the bias voltage. Furthermore, the noise floor rises with frequency and shows a marked increase above the sensor resonance. At first glance, this looks similar to the result of deliberate noise shaping that occurs in a $\Sigma \Delta$ system seen in in Figure 4a. In the EMPLL, however, this behaviour is not intentional and is merely an artefact from the combined sensor and loop filter transfer functions in the closed-loop system. Indeed, when a generic secondorder system with appropriately placed complex poles is used in the loop filter, this increase of noise at high frequencies can be significantly reduced.

\subsection{Input Amplitude Sweep}

One significant problem with $\operatorname{EM} \Sigma \Delta$ systems are stability issues for large input amplitudes, as can be seen in Figure 5. In this case, the 5th order system can typically cope with accelerations of up to $3 \mathrm{~g}$ and then the system will lose stability and become less able to detect the response of the sensor.

In contrast, the results of the EMPLL as shown in Figure 5 demonstrate a much wider potential range of accelerations possible to be sensed. In a first approximation, the EMPLL is a linear system, which means there are no fundamental restrictions on amplitude performance. There is a certain point 


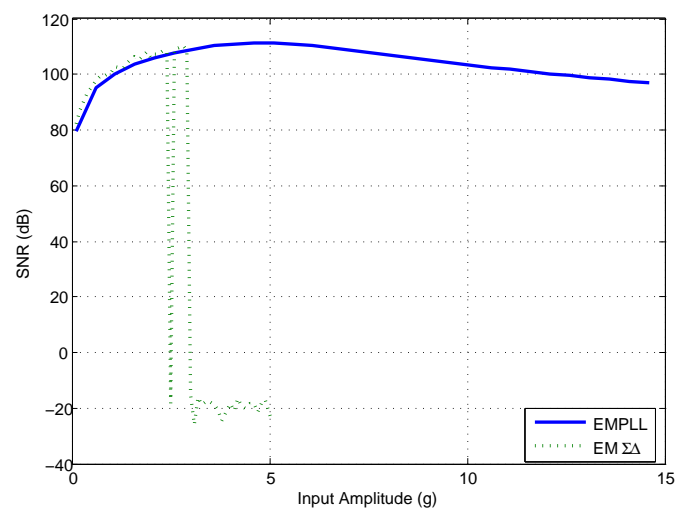

Figure 5: Input amplitude sweep to $15 \mathrm{~g}$ for EM $\mathrm{E} \Delta$ and EMPLL

beyond which further increase of the input amplitude causes the quadratic term from equation 12 to rise above the noise floor which leads to a reduction of overall SNR. It also has to be noted that there is a maximum input amplitude for the EMPLL, although for different technical reasons. In closed-loop control, the accelerometer can be subject to much larger accelerations than without feedback because the feedback force on the proof mass counteracts the force to acceleration. The feedback force, however, is limited in amplitude by the bias voltage. If the acceleration results in a force that is larger than can be compensated by the feedback force, the proof mass can no longer be controlled. Rearranging and evaluating equations 10 and 1 at the bias voltage, while ignoring displacement results in the expression in 15 for the maximum acceleration.

$$
a_{\text {max }}=2 \frac{k_{\text {force }}}{m} \frac{V_{\text {bias }}^{2}}{d_{\text {nom }}^{2}}
$$

The factor 2 in the equation is a result of there being two feedback system 
driven by a differential feedback voltage, exerting twice the force of a single system. Evaluating equation 15 for nominal sensor parameters and a bias voltage of $20 \mathrm{~V}$ results in a maximum acceleration of about $70 \mathrm{~g}$. Note that since the bias voltage influences this term quadratically, this figure drops significantly for lower bias voltages. At $12 \mathrm{~V}$ bias, for example, the maximum acceleration the system can handle is $26 \mathrm{~g}$.

\subsection{Parameter Variation}

In order to investigate the EMPLL and $\operatorname{EM} \Sigma \Delta$ circuit's susceptibility to device parameter variation, Monte Carlo simulations were performed based on the values given in Tables 2 and 3. The yield of $\operatorname{EM} \Sigma \Delta$ systems having a SNR of $90 \mathrm{~dB}$ or greater in 500 runs was $53.2 \%$. The SNR distribution of the $\operatorname{EM} \Sigma \Delta$ systems is shown in Figure 6a, with a mean SNR of 107.6dB. The standard deviation in SNR is $1.707 \mathrm{~dB}$, corresponding to $1.6 \%$ of the mean value. The yield of EMPLL systems having a SNR of $90 \mathrm{~dB}$ or greater in 500 runs was $89.2 \%$. The SNR distribution of the systems is shown in Figure $6 \mathrm{~b}$. It can be seen that the distribution is narrower than the one of the $\operatorname{EM} \Sigma \Delta$, having a standard deviation of $0.349 \mathrm{~dB}$, corresponding to $0.31 \%$ of the mean value.

This result is a significant improvement over $\Sigma \Delta$ systems, which are very susceptible to parameter variation. This can once again be attributed to the approximately linear system structure of the EMPLL. The histograms in Figure 6 show the effect of SNR degradation. 


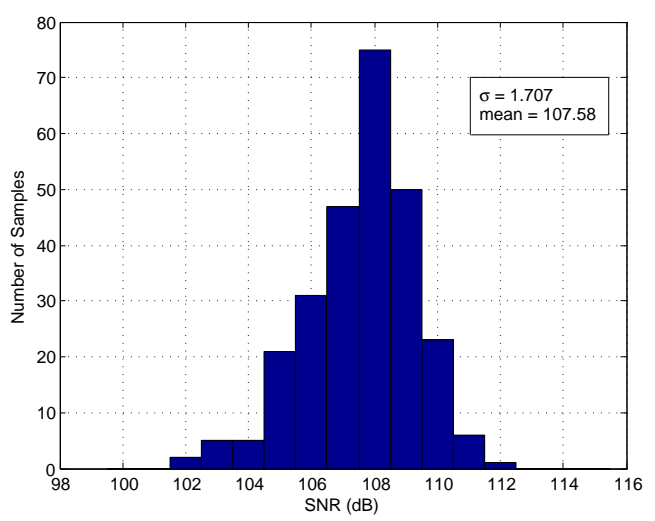

(a) $\operatorname{EM} \Sigma \Delta$

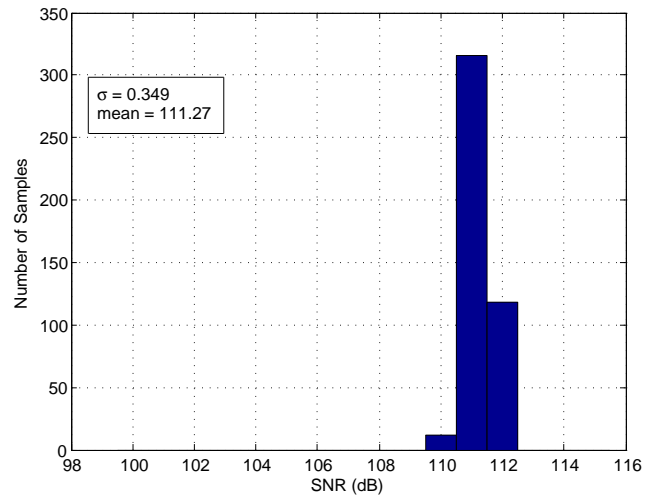

(b) EMPLL

Figure 6: SNR histograms of EM $\Sigma \Delta$ and EMPLL under parameter variation.

\subsection{Summary of Results}

The comparison with a 5 th order $\operatorname{EM} \Sigma \Delta$ System is useful, as this is a very typical system used in many applications. While it is true that each individual parameter could possibly be improved in the EM $\Sigma \Delta$ system, looking at all three aspects (SNR, Amplitude Range and Variation Tolerance), the EMPLL simulation results indicate an exciting alternative approach which is currently being tested practically.

\section{Conclusion}

In this paper we have described a novel differential frequency domain technique for closed loop control of micro-machined sensors. This method, called the electro-mechanical phase locked loop (EMPLL), uses a differential electro-mechanical phase locked loop to control and measure the deflection of MEMS sensors. Preliminary results indicate that EMPLLs have the potential to have significant advantages over $\operatorname{EM} \Sigma \Delta$ s for high performance MEMS 
sensors. In particular we have shown there are three areas where this novel approach will lead to significant benefits over previous approaches which are Signal to Noise Ratio, Parameter Sensitivity, and Input Signal Range.

Our tests have shown that for the same sensor, the EMPLL and a $5^{\text {th }}$ order $\operatorname{EM} \Sigma \Delta$ circuit both provide an SNR performance of around $110 \mathrm{~dB}$ for the same signal bandwidth, which demonstrate the same fundamental noise performance. A striking difference, however, is the ability of the EMPLL circuit to tolerate much greater levels of acceleration, with nearly $100 \mathrm{~dB}$ of SNR achieved up to nearly $15 \mathrm{~g}$, indicating a much higher tolerance than the equivalent $\operatorname{EM} \Sigma \Delta$ circuits. Finally, it is well known that the $\operatorname{EM} \Sigma \Delta$ circuits are extremely sensitive to parameter variations and the EMPLL circuits demonstrate an improved tolerance to those variations with an $80 \%$ reduction in variance of SNR of the EMPLL over the $\operatorname{EM} \Sigma \Delta$ circuit.

In summary, this paper has not only shown that the EMPLL approach can provide similar SNR performance to a conventional $\operatorname{EM} \Sigma \Delta$, but that in addition it has the benefit of a much wider range of input acceleration for an identical sensor and also that the circuit offers a very robust system that is tolerant to variations in both the mechanical parts of the sensor, but also the electronic circuit.

\section{References}

[1] M. K. X. Jiang, J. Seeger, B. Boser, A monolithic surface micromachined z-axis gyroscope with digital output, in: Symposium on VLSI Circuits, Hawaii, USA, 2000, pp. 16-19. 
[2] C. L. M. Kraft, T. Hesketh, Closed loop silicon accelerometers, IEE Proceedings Circuits, Devices and Systems.

[3] Y. Dong, M. Kraft, C. O. Gollasch, A high performance accelerometer with fifth order sigma delta modulator, J Micromech Microeng 15 (2005) $1-8$.

[4] J. Luo, H. Ding, M. Kraft, A new design methodology for electromechanical sigma- delta-modulators, in: Nano/Micro Engineered and Molecular Systems, 2009. NEMS 2009. 4th IEEE International Conference on, 2009, pp. $881-884$.

[5] R. Wilcock, M. Kraft, Genetic algorithm for the design of electromechanical sigma delta modulator mems sensors, Sensors.

[6] Y. Matsumoto, M. Nishimura, M. Matsuura, M. Ishida, Three-axis soi capacitive accelerometer with pll c-v converter, Sensors and Actuators A: Physical 75 (1) (1999) $77-85$.

[7] Y. Matsumoto, M. Esashi, Integrated silicon capacitive accelerometer with pll servo technique, Sensors and Actuators A: Physical 39 (3) (1993) $209-217$.

[8] T. M. Mayo Kitano, Hideki Sumi, Capacitance difference detecting circuit and mems sensor, Patent (US 7119550).

[9] M. Saukoski, L. Aaltonen, T. Salo, K. A. Halonen, Interface and control electronics for a bulk micromachined capacitive gyroscope, Sensors and Actuators A: Physical 147 (1) (2008) 183 - 193. 
[10] R. Fang, W. Lu, C. Liu, Z. Chen, Y. Ju, G. Wang, L. Ji, D. Yu, A lownoise interface circuit for mems vibratory gyroscope, in: Solid-State and Integrated Circuit Technology (ICSICT), 2010 10th IEEE International Conference on, 2010, pp. $1456-1458$.

[11] M. L. Aaron Partridge, Frequency and/or phase compensated microelectromechanical oscillator, Patent (US 6995622).

[12] C. W. N. A. Hati, D. A. Howe, Phase-lock loops in vibration environments, in: 41st Annual Precise Time and Time Interval (PTTI) Meeting, 2009.

[13] S. Yoneoka, J. Salvia, G. Bahl, R. Melamud, S. Chandorkar, T. Kenny, Active electrostatic compensation of micromechanical resonators under random vibrations, Microelectromechanical Systems, Journal of 19 (5) (2010) 1270 -1272. doi:10.1109/JMEMS.2010.2067444. 